\title{
Apoptosis induced by PGC-1 $\beta$ in breast cancer cells is mediated by the mTOR pathway
}

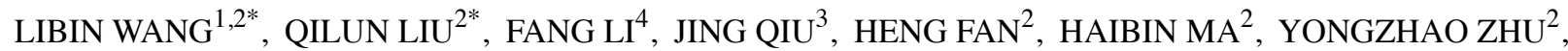 \\ LIGANG WU $^{2}$, XUEBO HAN ${ }^{2}$, ZHIHONG YANG ${ }^{2}$, HAIFENG JIANG $^{2}$, JUN WEI $^{2}$ and HAIBIN XIA ${ }^{1}$ \\ ${ }^{1}$ Life Science College, Shaanxi Normal University, Xi'an 710062; ${ }^{2}$ Ningxia Human Stem Cells Institute of General Hospital, \\ Fertility Conservation Laboratory of Ministry of Education, Ningxia Medical University, Yinchuan 750004; \\ ${ }^{3}$ Department of Stomatology, Qingdao Municipal Hospital, Qingdao 266000; ${ }^{4}$ Maternity and Child \\ Health Hospital of Ningxia Hui Autonomous Region, Yinchuan 750004, P.R. China
}

Received February 17, 2013; Accepted April 19, 2013

DOI: $10.3892 /$ or.2013.2628

\begin{abstract}
The peroxisome proliferator-activated receptor- $\gamma$ (PPAR- $\gamma$ ) coactivator-1 $\beta$ (PGC-1 $\beta$ ) is a well-established regulator of mitochondrial biogenesis. However, the underlying mechanism of PGC- $1 \beta$ action remains elusive. This study reveals that knockdown of endogenous PGC-1 $\beta$ by shorthairpin RNA (shRNA) leads to a decrease in the expression of mammalian target of rapamycin (mTOR) pathway-related genes in MDA-MB-231 cells. After knockdown of PGC-1 $\beta$, phosphorylation of AMP-activated protein kinase (AMPK), phosphorylation of Rictor on Thr1135, Raptor and S6 protein was inhibited. However, Akt phosphorylation on Ser473 was upregulated and cell apoptosis occurred. In particular, we demonstrate that the levels of PGC- $1 \beta$ and mTOR correlated with overall mitochondrial activity. These results provide new evidence that cell apoptosis is orchestrated by the balance between several signaling pathways, and that PGC-1 $\beta$ takes part in these events in breast cancer cells mediated by the mTOR signaling pathway.
\end{abstract}

\section{Introduction}

Breast cancer, a complex and heterogeneous disease, is a leading cause of cancer death in women. Each year, there are

Correspondence to: Professor Haibin Xia, Life Science College, Shaanxi Normal University, 199 South Chang'an Street, Xi'an 710062, P.R. China

E-mail: hbxia2001@yahoo.com

Professor Jun Wei, Ningxia Human Stem Cells Institute of General Hospital, Fertility Conservation Laboratory of Ministry of Education, Ningxia Medical University, Yinchuan 750004, P.R. China

E-mail: lydiajunwei@hotmail.com

*Contributed equally

Key words: peroxisome proliferator-activated receptor-gamma coactivator-1 $\beta$, MDA-MB-231, mammalian target of rapamycin pathway, apoptosis over 1.5 million newly diagnosed cases, and 500,000 women worldwide die of this disease (1). Despite combined treatment with surgery, radiotherapy, and anticancer drugs, many breast cancer patients will ultimately develop metastatic disease, at present incurable (2). Various epidemiological studies have revealed that multiple factors including hormones, genetics, reproductive history, radiation, socio-economic status, place of residence, ethnicity, and the environment affect the incidence of breast cancer (3). The main therapeutic principles are to find the cellular signaling regulator of the breast cancer. Cellular signaling that govern cell proliferation, motility and survival are often deregulated in cancer cells (4-6). Such regulation is managed by signaling pathways acting through transcription factors that control expression of specialized genes and their protein products to control the differentiation as well as bioenergetic capacity of cancer cells $(7,8)$.

Of these signaling pathways, increasing attention has been placed on mammalian target of rapamycin (mTOR) pathway, which is recognized as serine/threonine protein kinase important for cellular growth, proliferation, motility and survival (9). It belongs to the family of phosphatidylinositol 3-kinase-related kinases, and dysregulation is associated with various diseases including several malignancies (10).

Mitochondrial biogenesis is regulated by a set of transcription factors that include nuclear respiratory factor 1 (NRF1) and NRF2, mitochondrial transcription factor A (mtTFA), the peroxisome proliferator-activated receptors (PPARs) coactivator $1 \alpha$ (PGC- $1 \alpha)$ and PGC- $1 \beta$ transcriptional coactivators $(11,12)$. PGC-1 proteins additionally serve as mediators between various external physiological stress conditions that enhance mitochondrial activity as well as drive the formation of slow type I myofibers and their associated oxidative metabolic phenotype (13). Relatively little is known about what controls PGC-1 gene expression or other mitochondrial regulators, although some myogenic factors have been identified $(14,15)$. The nutrient sensor, mTOR, was also shown to promote mitochondrial oxidative function through transcriptional control of PGC-1 $(16,17)$.

In the present study, we hypothesized that PGC-1 $\beta$ has a critical role in breast cancer cell growth through regulation of the mTOR signaling pathway. To test this hypothesis, 
short-hairpin RNA (shRNA)-specific PGC-1 $\beta$ was generated and the expression of mTOR pathway-related genes in MDA-MB-231 cells was investigated. Consistent with this hypothesis, phosphorylation of AMP-activated protein kinase (AMPK), phosphorylation of Rictor on Thr1135, Raptor and S6 protein is inhibited. Additionally, Akt phosphorylation on Ser473 is upregulated and cell apoptosis by PGC-1 $\beta$ occurs. These findings provide novel insights into how breast cancer cells are protected and highlight the importance of PGC-1 $\beta$ in regulation of breast cancer cell apoptosis.

\section{Materials and methods}

Plasmids. pcDNA3.1-PGC-1 $\beta$ for the expression of human full-length PGC-1 $\beta$ was a gift from Ling Wang (Peking Union Medical College, China). Adenoviral vectors expressing green fluorescent protein (GFP), PGC-1 $\alpha$ or ERR $\alpha$ were generated using the AdEasy system. The pGenesil vector expressing shRNAs against PGC-1 $\beta$ (shPGC-1 $\beta$ ) was constructed by our laboratory, targeting the sequence $5^{\prime}$-ttgtacagaactacataagcac-3' of human PGC-1 $\beta$. All plasmid constructs were verified by sequencing.

Cell culture. Human embryonic kidney 293 (HEK293) cells, from Microbix Biopharmaceuticals (Toronto, ON, Canada) and human breast cancer cells MDA-MB-231 from American Type Culture Collection (ATCC, Manassas, VA, USA) were cultured in Dulbecco's modified Eagle's medium (DMEM) supplemented with $10 \%$ fetal bovine serum (FBS), $2 \mathrm{mM}$ L-glutamine, $100 \mathrm{U} / \mathrm{ml}$ penicillin, and $100 \mu \mathrm{g} / \mathrm{ml}$ streptomycin in a humidified atmosphere of $5 \% \mathrm{CO}_{2}$ at $37^{\circ} \mathrm{C}$.

Immunohistochemistry (IHC) analysis. The study population consists of the carcinoma of breast treated between July, 2008 and March, 2012 at General Hospital of Ningxia Medical University which are the main hospitals for the treatment of breast cancer in Yinchuan, China. IHC study was performed on paraffin-embedded, formalin-fixed tissue sections using a commercial available Ultra Sensitive ${ }^{\mathrm{TM}}$ SP kit (Santa Cruz Biotechnology, Inc., Santa Cruz, CA, USA), following the manufacturer's instructions and the guideline recommendations for human PGC-1 $\beta$ testing in breast cancer. Briefly, this procedure included the deparaffinization and rehydration steps, followed by an epitope retrieval step in which the tissue sample was incubated in a citrate buffer solution at $90-95^{\circ} \mathrm{C}$ for $20 \mathrm{~min}$. The slides were then subjected to a series of alternating washes in PBS buffer and incubation steps with, first, a peroxidase-blocking reagent for $5 \mathrm{~min}$ and then with PGC-1 $\beta$ primary antibody, followed by a visualization reagent for $30 \mathrm{~min}$ each, and finally with a 3,3'-diaminobenzidine chromogen solution. After a final wash, the slides were counterstained with haematoxylin.

RNA isolation and quantitative real-time PCR (qRT-PCR). Total RNA from cultured cells was extracted using TRIzol reagent (Invitrogen). cDNA was then reverse-transcribed and amplified by PCR using a Transcriptor Reverse Transcriptase kit (Roche). qRT-PCR was carried out using the SYBR-Green PCR system on a Bio-Rad iQ5, and results were normalized to glyceraldehyde-3-phosphate dehydrogenase (GAPDH) or cyclophilin A. The sequences of primers are shown in Table I.

Western blot analysis. After treatments, cells were lysed by the addition of cold RIPA buffer [ $150 \mathrm{mM} \mathrm{NaCl}, 50 \mathrm{mM}$ Tris $\mathrm{HCl}$, $0.1 \%$ SDS, $1 \%$ Triton X-100, $1 \mathrm{mM}$ PMSF, $2 \mathrm{mM} \mathrm{NaF}, \mathrm{Na}_{3} \mathrm{VO}_{4}$, $\beta$-glycerophosphate and $2 \mathrm{mM}$ EDTA, and fresh protease inhibitor cocktail (Sigma Aldrich)], and cell lysate was centrifuged at $14,000 \mathrm{x} \mathrm{g}$ at $4^{\circ} \mathrm{C}$ for $20 \mathrm{~min}$. The supernatant was harvested and analyzed for protein content using protein assay dye. Protein was denatured in sample buffer, then separated on SDS-PAGE, and transferred to polyvinylidene difluoride (PVDF) membranes using a semi-dry Trans-Blot system. The blots were blocked for $1 \mathrm{~h}$ at room temperature with Tris-buffered saline (TBS, $50 \mathrm{mM}$ Tris- $\mathrm{HCl}, \mathrm{pH} 7.5,150 \mathrm{mM}$ $\mathrm{NaCl}$ ) containing $5 \%$ non-fat milk. The blots were washed three times with TBST (50 mM Tris- $\mathrm{HCl}, \mathrm{pH} 7.5,150 \mathrm{mM}$ $\mathrm{NaCl}$ and $0.02 \%$ Tween-20) and incubated with the indicated primary antibody at $4^{\circ} \mathrm{C}$ overnight. The next day, the blots were incubated for $1 \mathrm{~h}$ at room temperature with secondary antibody (1:5,000 dilutions), and detected by ECL detection reagent. To ensure that equal amounts of sample protein were applied for electrophoresis, GAPDH or the indicated proteins were used as an internal control.

Cells were pretreated for the AMP and ATP measurements. The AMP and ATP expression level was measured. Briefly, culture medium was removed, cells were washed with ice-cold PBS, and $500 \mathrm{ml}$ of ice-cold $0.4 \mathrm{M}$ perchloric acid was added. The culture dishes were cooled to $-80^{\circ} \mathrm{C}$ then cell lysates were thawed on ice, scraped off, and transferred to microfuge tubes. Samples were centrifuged at $15,000 \mathrm{xg}$ for $10 \mathrm{~min}$ at $4^{\circ} \mathrm{C}$. The supernatant was neutralized with $50 \mathrm{ml} 4 \mathrm{M} \mathrm{K}_{2} \mathrm{CO}_{3}$, kept on ice for $10 \mathrm{~min}$, and placed at $-80^{\circ} \mathrm{C}$ for $2 \mathrm{~h}$ to allow precipitation of the perchlorate. Samples were centrifuged again and supernatants were kept at $-80^{\circ} \mathrm{C}$ until nucleotide measurements.

AMP determination by HPLC. For the quantification of AMP present in MDA-MB-231 cell extracts, the nucleotides were separated by HPLC using a SynChropak AX100 anion exchange column (Phenomenex). The nucleotides were eluted isocratically at ambient temperature with a mobile phase of $125 \mathrm{mM} \mathrm{K}_{2} \mathrm{HPO}_{4}, 0.5 \mathrm{M} \mathrm{KCl}$, adjusted to $\mathrm{pH} 6.0$ with $5 \mathrm{~N}$ $\mathrm{NaOH}$. Forty microliters of nucleotide cell extracts described above were diluted to $100 \mathrm{ml}$ in the mobile phase and $50 \mathrm{ml}$ were injected into the instrument. Absorbance was monitored at $260 \mathrm{~nm}$ and retention time for AMP was $7.0 \mathrm{~min}$. The amount of AMP contained in each fraction was measured by comparing the height of the peak with a standard curve of AMP, ranging from 0 to 40 pmoles.

ATP determination by fluorometric assay kit. ATP quantification was performed using the Biovision ATP Colorimetric/ Fluorometric Assay kit (Cedarlane). Briefly, the fluorometric assay was performed as described by the manufacturer, using a standard curve of 0 to 200 pmoles. Ten microliters of nucleotide extract, diluted to $50 \mathrm{ml}$ with ATP assay buffer, was used for each quantification. Plates were incubated in the dark for $30 \mathrm{~min}$. Data were acquired on a CytoFluor ${ }^{\mathrm{TM}} 2350$ (Millipore) using black 96-well assay plates. ATP was quanti- 
Table I. Primers of hCD40L.

\begin{tabular}{lll}
\hline Primer & \multicolumn{1}{c}{ Forward (5'-3') } & \multicolumn{1}{c}{ Reverse (5'-3') } \\
\hline COX I & GGATTTGTTCACTGATTCCCATT & CATCTGGGTAGTCTGAGTAGCG \\
COX II & AGGCCGACTAAATCAAGCAAC & CTAGGACAAT GGGCATAAAG \\
COX VIIa & ATGAGGGCCCTACGGGTCT & CATTGTCGGCCTGGAAGAG \\
Cyclophilin A & TTCCTCCTTTCACAGAATTATTCC & CCGCCAGTGCCATTATGG \\
GAPDH & GGAGCGAGATCCCTCCAAAAT & GGCTGTTGTCATACTTCTCATGG
\end{tabular}

COX, cytochrome c oxidase; GAPDH, glyceraldehyde-3-phosphate dehydrogenase.

fied by comparing the absorbance values obtained at $590 \mathrm{~nm}$ with those of the standard curve.

Cell transfection and microscopy analysis. Cells were grown on coverslips in a 6-well plate and transfected with $0.5 \mathrm{mg}$ of the GFP-LC3 expression plasmid using Lipofectamine ${ }^{\mathrm{TM}} 2000$ (Invitrogen) according to the manufacture. After treatment, as defined in the figure legends, cells were washed with PBS and fixed with $4 \%$ formaldehyde in PBS for $15 \mathrm{~min}$ at room temperature. Coverslips were inverted on a drop of Fluoromount-G (SouthernBiotech). Transfected cells were observed and images were taken using a fluorescence microscope (Leica Microsystems).

Detection of cell apoptosis by flow cytometry. Cells $\left(1 \times 10^{5}\right)$ were washed with PBS and incubated in a solution of $0.5 \mathrm{~g} / \mathrm{ml}$ FITC-labeled Annexin V for $10 \mathrm{~min}$, washed with PBS and stained by $10 \mu \mathrm{l}$ of $20 \mu \mathrm{g} / \mathrm{ml}$ propidium iodide (PI) for $10 \mathrm{~min}$, add $190 \mu \mathrm{l}$ binding buffer, all the step following Annexin V-FITC Apoptosis Detection kit I (BD Biosciences). Cells were then analysis by flow cytometry (FACSCalibur; BD Biosciences), and CellQuest software. Ten thousand events are acquired for statistical analysis.

Statistical analysis. All the results were expressed as mean \pm standard error of the mean (SEM). All statistical analysis was evaluated using GraphPad Prism software (GraphPad Software, Inc., San Diego, CA, USA). Data were analyzed by the paired t-test, and P-values $<0.05$ was considered statistically significant.

\section{Results}

PGC-1 $\beta$ is overexpressed in human breast cancer tissue vs. normal tissue. We determined the expression pattern of PGC-1 $\beta$ in clinical human breast normal and cancer samples. Compared to the normal tissue, the expression of PGC- $1 \beta$ in tumor samples was mostly increased. We found that breast cancer samples showed strong immunostaining of PGC-1 $\beta$ compared to normal breast cells, representative images of both breast cancer and normal are shown in Fig. 1. PGC-1 $\beta$ was significantly overexpressed in breast cancer.

The effects of PGC-1 $\beta$ on intracellular mitochondrial biogenesis. PGC-1 is suggested to be a potent stimulator of mitochondrial biogenesis $(18,19)$. We evaluated the role of
PGC-1 on mitochondrial biogenesis. To measure mitochondrial DNA directly, we isolated total DNA and determined the relative copy number of mitochondrial DNA by a qPCR assay of the mitochondrial DNA-encoded cytochrome $\mathrm{c}$ oxidase (COX) II gene. Overexpression of PGC-1 $\beta$ led to an increase in mitochondrial DNA content/cell by 1.97 -fold (Fig. 2A), while inhibition of endogenous PGC-1 $\beta$ by shRNA changed the basal mitochondrial DNA content (Fig. 2A). Furthermore, knockdown of endogenous PGC-1 $\beta$ by shRNA reduced the induction of mitochondrial biogenesis. In addition, mRNA transcript levels of COX I and COX II (mitochondrialencoded) and COX VIIa (nuclear-encoded) were induced by PGC-1 $\beta$ (Fig. 2B). Knockdown of endogenous PGC-1 $\beta$ blocked the effect of COX I, COX II and COX VIIal gene expression (Fig. 2B). These results reveal the important physiological role of PGC-1 $\beta$ in mitochondrial biogenesis.

PGC-1 $\beta$ affects AMPK activation and mTORC1/2 complexes. It is well documented that an increase in AMP/ATP ratio is the key signal to activate AMPK in an attempt to preserve cellular energy $(20,21)$. In order to evaluate the energetic status of cells, we treated MB-MD-231 cells with pcDNA3PGC-1 $\beta$ or pGenesil-shPGC-1 $\beta$, then measured the levels of ATP and AMP after treatment for $1 \mathrm{~h}$. The drastic increase in AMP levels was observed after treatment pcDNA3-PGC-1 $\beta$ for $1 \mathrm{~h}$ (Fig. 3A). We also observed a drastic decrease in ATP levels $1 \mathrm{~h}$ following pcDNA3-PGC-1 $\beta$ transfection (Fig. 3B). However, the AMP and ATP remained at control levels when cells were treated with control vector (Fig. 3A and B). The variation in ATP and AMP levels in response to pcDNA3PGC-1 $\beta$ treatment is illustrated with the AMP/ATP ratio (Fig. 3C). We observed a significant increase in the AMP/ATP ratio following pcDNA3-PGC-1 $\beta$ exposure for $1 \mathrm{~h}(\mathrm{P}<0.05)$. To further validate the role of $\mathrm{PGC}-1 \beta$ in regulating AMP and ATP expression, RNA interference was employed to knockdown the endogenous PGC-1 $\beta$ expression in MDA-MB-231 cells. AMP level, AMP/ATP ratio was downregulated and ATP level upregulated when PGC- $1 \beta$ expression and activity were suppressed with pGenesil-shPGC-1 $\beta$. We observed no change in the AMP/ATP ratio when cells were treated with the control vector (Fig. 3).

AMPK, the energy sensor of the cell, is a heterotrimeric Ser/Thr protein kinase activated by alterations in cellular AMP/ATP ratio (22). In order to determine whether the increase in AMP/ATP ratio, caused by PGC-1 $\beta$ upregulation, leads to the activation of AMPK, we analyzed the phos- 

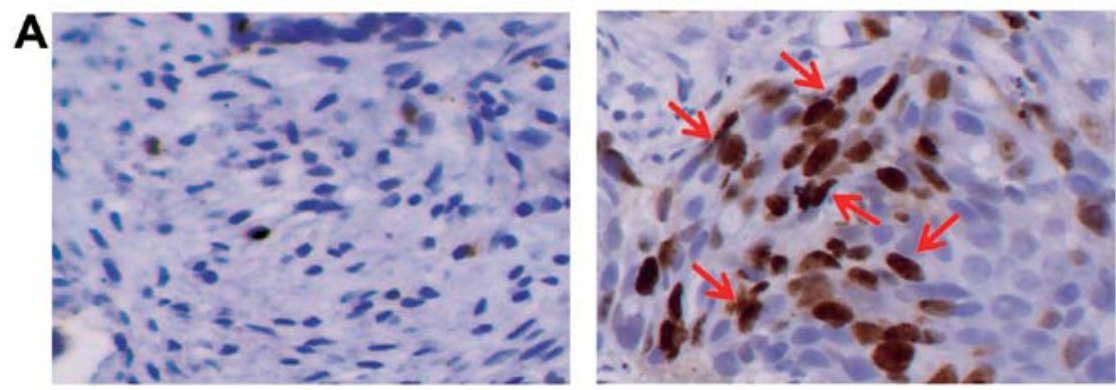

B

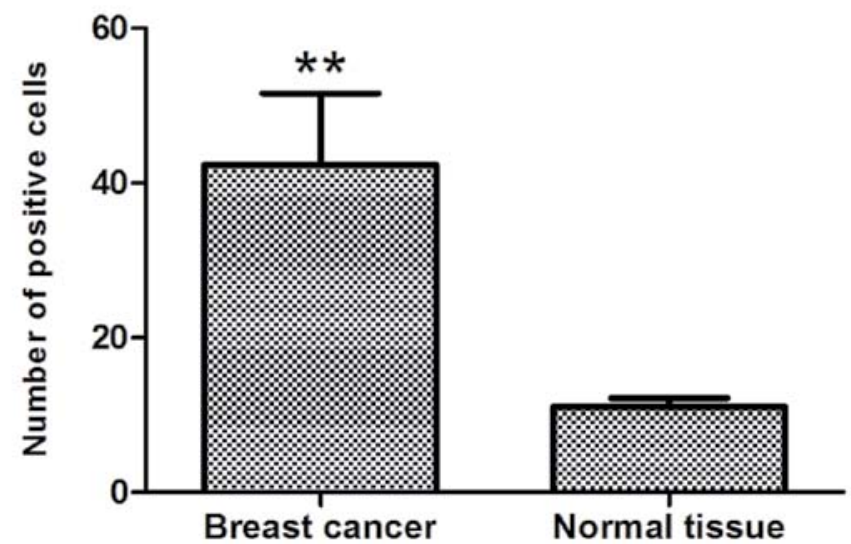

Figure 1. Peroxisome proliferator-activated receptors coactivator $1 \beta$ (PGC-1 $\beta$ ) is overexpressed in human breast cancer tissues compared to normal tissue samples. (A) Immunohistochemical staining of PGC-1 $\beta$. The tissue was stained for PGC-1 $\beta$. (B) Quantitation of PGC-1 $\beta$ immunostaining. Numbers of positive cells were counted for PGC-1 $\beta$ staining. Tissue types were grouped. The groups were compared using the two-tailed Fisher's exact test with a ${ }^{* *} \mathrm{P}$-value of 0.05 and was therefore considered statistically significant. Red arrowhead stands for the positive PGC-1 $\beta$ staining.

A

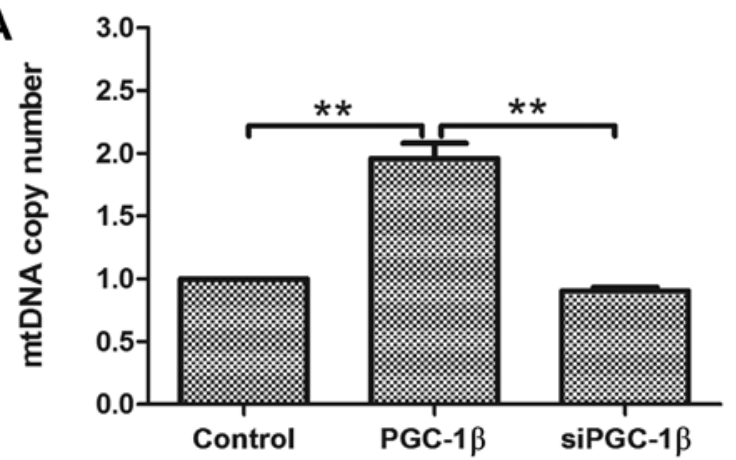

B

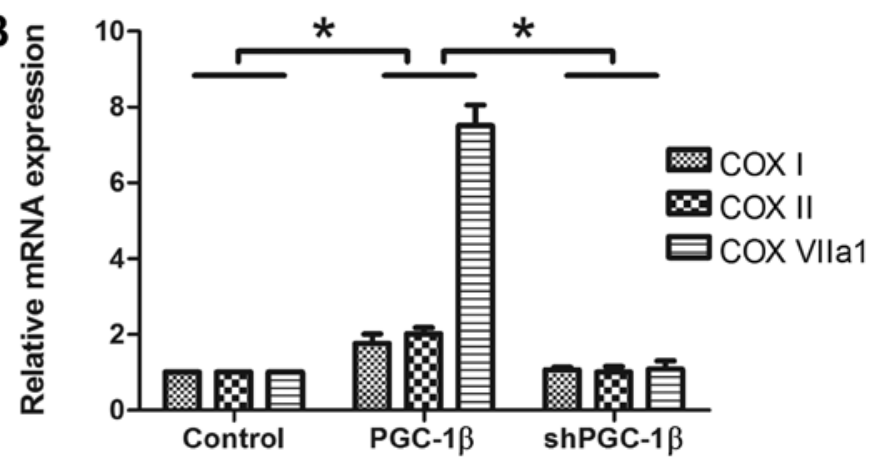

Figure 2. Evaluation of peroxisome proliferator-activated receptors coactivator $1 \beta$ (PGC-1 $\beta$ ) on the mitochondrial biogenesis. (A) PGC-1 $\beta$ downregultion stimulated mitochondrial biogenesis. MDA-MB-231 were transfected with pcDNA3-PGC-1 $\beta$ or pGenesil-shPGC-1 $\beta$. Cells were harvested $48 \mathrm{~h}$ after transfection, and the DNA level of the mitochondrial-encoded cytochrome c oxidase (COX) II gene was measured by quantitative PCR (qPCR), normalized to DNA levels of the nuclear-encoded gene cyclophilin A, and expressed relative to levels in control cells, which were set to 1. (B) Knockdown of PGC-1 $\beta$ inhibited the

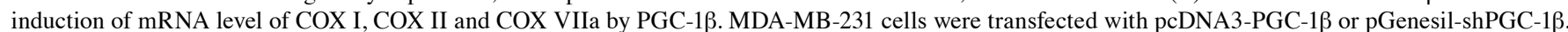
Cells were harvested and total RNA was extracted $48 \mathrm{~h}$ after transfection. The mRNA levels of mitochondrial-encoded (COX I and COX II) and nuclearencoded (COX VIIa) subunits of COX were measured by qPCR, normalized to glyceraldehyde-3-phosphate dehydrogenase (GAPDH), and expressed relative to control cells. Values represent the mean of three independent experiments performed in duplicate. ${ }^{*} \mathrm{P}<0.05$ and ${ }^{* *} \mathrm{P}<0.01$.

phorylation levels of AMPK on Thr172 by immunoblotting. We observed a significant activation of AMPK for $12 \mathrm{~h}$, after pcDNA3-PGC-1 $\beta$ treatment in MD-MB-231 cells (Fig. 4A). The activation of AMPK is coupled with an increase in the cellular AMP/ATP ratio (Fig. 3C). Once AMPK activated, the AMPK inhibits ATP consuming anabolic processes such as protein translation.

mTOR encompasses two functionally distinct protein complexes: mTOR complex 1 and complex 2. The mTORC1 consists of mTOR, raptor, mLST8, and two negative regula- tors, PRAS40 and DEPTOR, Raptor being a direct substrate of activated AMPK, regulates mTOR activity and functions as a scaffold for recruiting mTORC1 substrates (23). Since AMPK activation achieves these effects largely through inhibition of mTOR signaling (24). We observed concomitant phosphorylation of the mTORC1 component Raptor on Ser792 (Fig. 4C). Then, we observed a drastic increase in the phosphorylation of the mTORC1 target S6 ribosomal protein, following pcDNA3-PGC-1 $\beta$ exposure (Fig. 4E). Furthermore, we observed a significant decrease in the phosphorylation of 

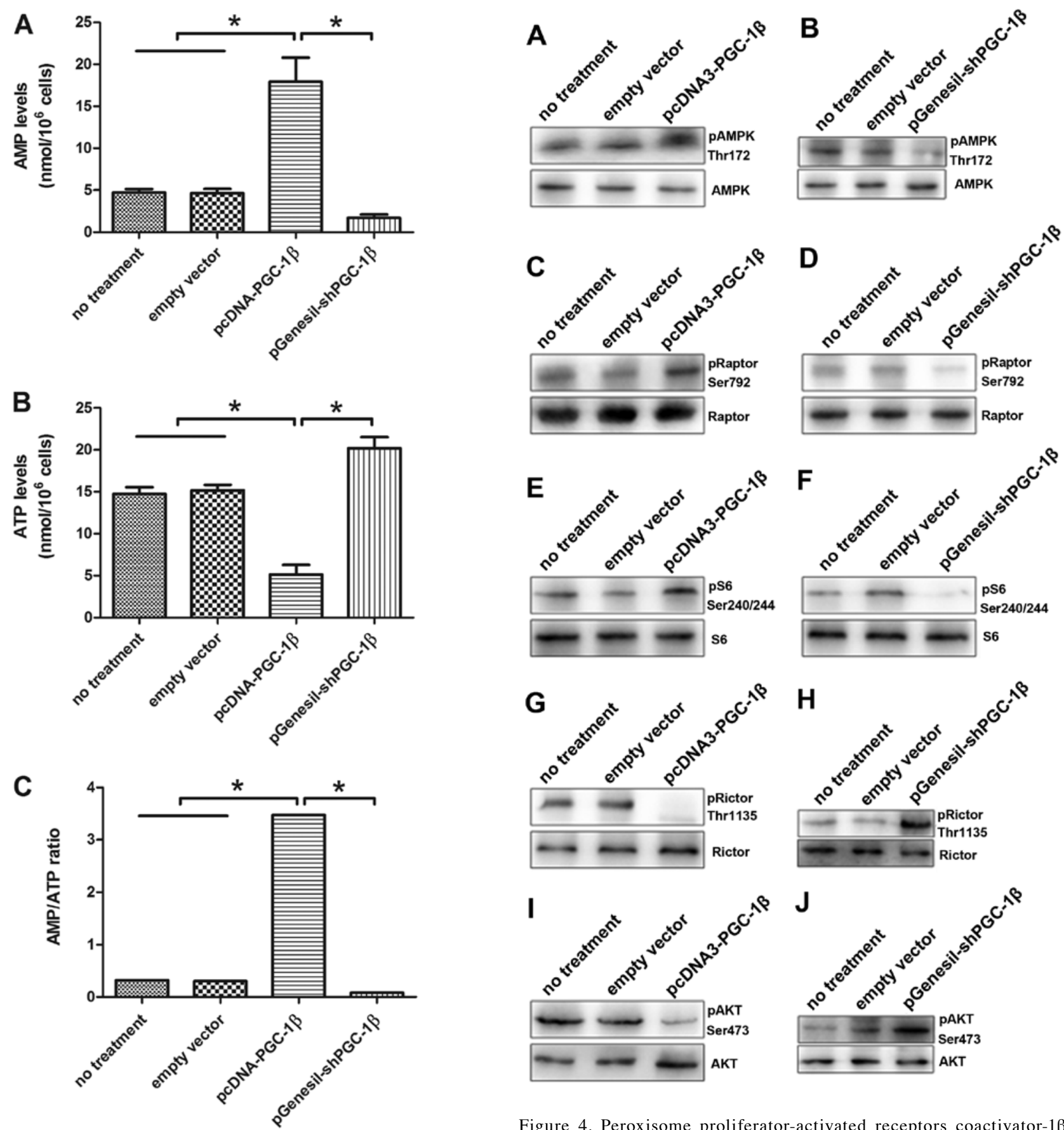

Figure 3. Peroxisome proliferator-activated receptors coactivator-1 $\beta$ (PGC-1 $\beta$ ) affects AMP and ATP levels. MDA-MB-231 cells were treated with empty vector, pcDNA3-PGC-1 $\beta$ or pGenesil-shPGC-1 $\beta$ and monitored for $1 \mathrm{~h}$. (A) AMP levels and (B) ATP levels were measured in MDA-MB-231 cells treated with empty vector, pcDNA3-PGC-1 $\beta$ or pGenesil-shPGC-1 $\beta$. (C) AMP/ATP ratios were calculated from AMP and ATP values at $1 \mathrm{~h}$. AMP and ATP levels were measured as described in Materials and methods. Data are presented as the mean \pm standard error of the mean of four independent experiments.
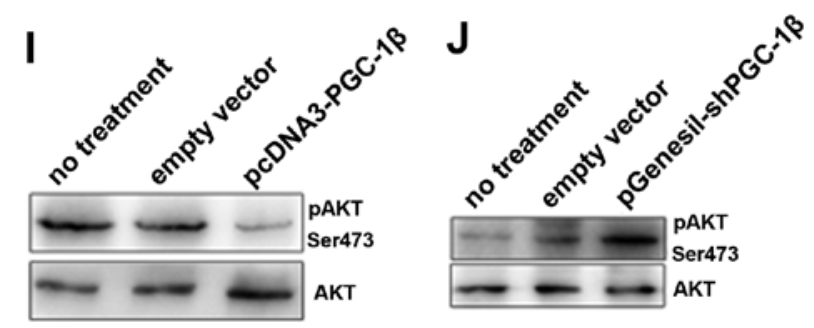

Figure 4. Peroxisome proliferator-activated receptors coactivator-1 $\beta$ (PGC-1 $\beta$ ) is associated with AMP-activated protein kinase (AMPK) activation and modulation of mTORC1/2 signaling. MDA-MB-231 cells were transfected with pcDNA3-P PGC-1 $\beta$, pGenesil-shPGC-1 $\beta$, empty vector alone. Cell lysates were analyzed by immunoblotting (A and B) for AMPK phosphorylation on Thr172, (C and D) Raptor phosphorylation on Ser792, (E and F) S6 phosphorylation on Ser240/244, (G and H) Rictor phosphorylation on Thr1135, and (I and J) Akt phosphorylation on Ser473. Total protein (AMPK, Raptor, S6, Rictor and Akt) was measured as an internal control (lower panels). Immunoblots are representative of three independent experiments.

Rictor on Thr1135, which is a key component of the mTORC2 complex (Fig. 4G). In MDA-MB-231 cells exposed to pGenesil-shPGC-1 $\beta$, the levels of AMPK and phosphorylation of Raptor were downregulated (Fig. 4D), while phosphorylation of S6 and Rictor was reversed (Fig. 4F and H), indicating that these events are specifically triggered by PGC-1 $\beta$ activation. Interestingly, we observed a significant downregulation

of Akt phosphorylation at Ser473 after pcDNA3-PGC-1 $\beta$ exposure (Fig. 4I), while inhibition of PGC-1 $\beta$ activation by pGenesil-shPGC-1 $\beta$ treatment led to a sustained and significant activation of Akt phosphorylation (Fig. 4J).

In summary, these data suggest that the PGC-1 $\beta$-dependent AMP accumulation following pcDNA3-PGC-1 $\beta$ exposure 
A

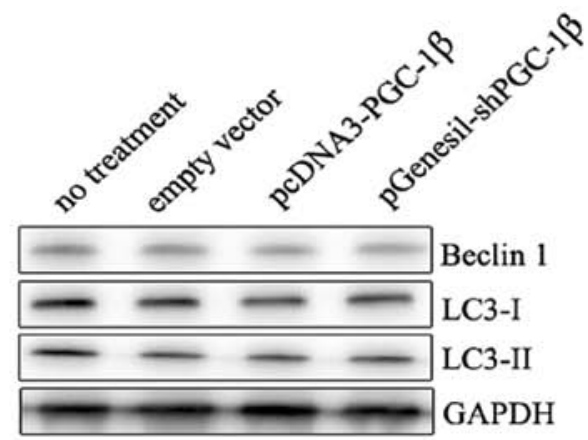

B

no treatment

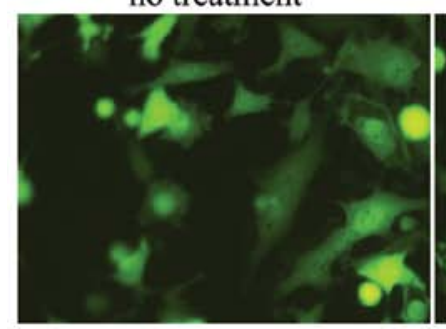

empty vector

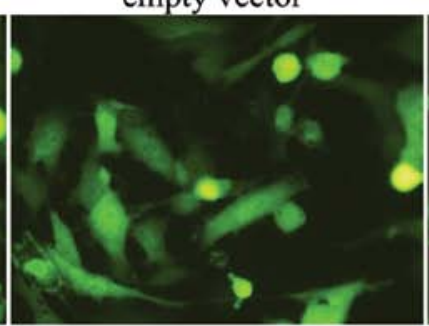

pcDNA3-PGC-1 $\beta$

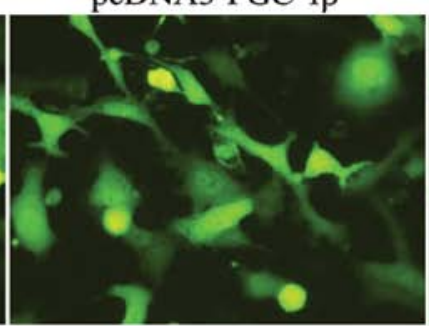

pGenesil-shPGC-1 $\beta$

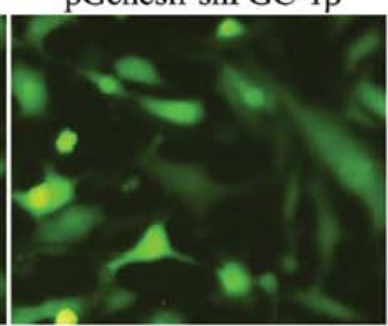

Figure 5. Effect of PGC-1 $\beta$ on Beclin 1 expression and on the autophagic marker LC3. MDA-MB-231 cells were transfected with pcDNA3-PGC-1 $\beta$, pGenesilshPGC-1 $\beta$, empty vector alone. (A) Beclin 1 expression and the conversion of LC3-I to LC3-II were detected by immunoblotting. The blots were also probed with glyceraldehyde-3-phosphate dehydrogenase (GAPDH) antibody to show equal loading. (B) Cells were transiently transfected with the green fluorescent protein (GFP)-LC3 plasmid for $24 \mathrm{~h}$ and in combination with pcDNA3-P PGC-1 $\beta$, pGenesil-shPGC-1 $\beta$ or empty vector. The distribution of GFP-LC3 was examined by fluorescence microscopy $24 \mathrm{~h}$ after the transfection and representative cells were photographed.

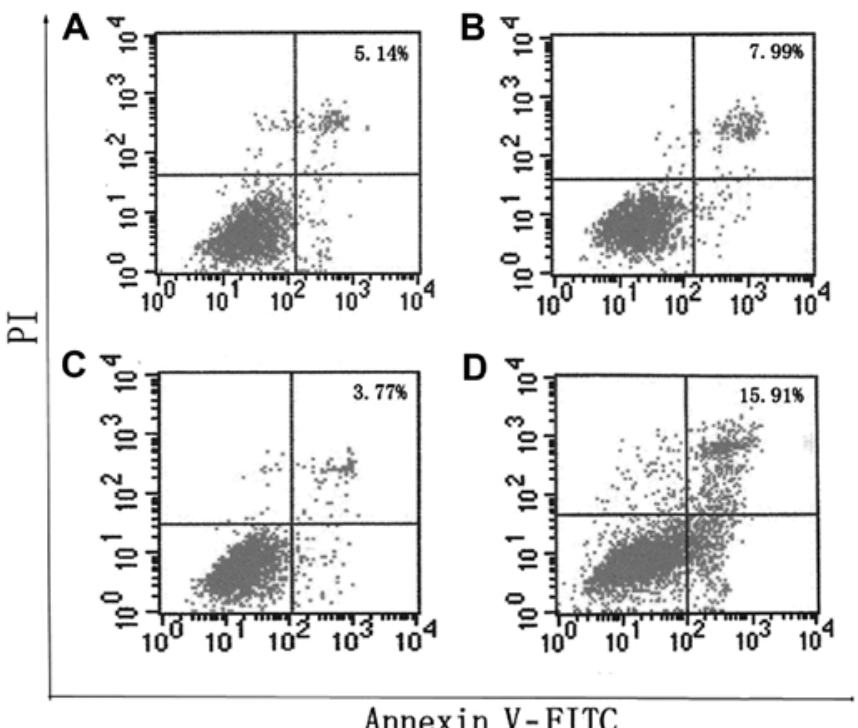

Annexin V-FITC

Figure 6. Effect of PGC-1 $\beta$ on cell apoptosis. MDA-MB-231 cells were transfected with (A) control, (B) empty vector, (C) pcDNA3-PGC-1 $\beta$, and (D) pGenesil-shPGC-1 $\beta$. Cell apoptosis was evaluated by staining with propidium iodide (PI) and Annexin V-FITC coupled with flow cytometry. Data are presented as the mean \pm SEM of at least three independent experiments.

leads to AMPK activation which affects some components of the $\mathrm{mTORC} 1 / 2$ signaling pathways.

Effect of PGC-1 $\beta$ activation on Beclin 1 expression and on the autophagic marker LC3. The mTOR pathway regulates cell autophagy under stress conditions, especially response to anticancer agents. Since we observed that PGC-1 $\beta$ down- regulation resulted in the suppression of mTORC1 signaling, we verified whether autophagy was subsequently induced. The conversion of LC3-I to LC3-II, a marker for autophagic vesicles and autophagy activity (25), was analyzed by immunoblotting. Unexpectedly, we did not observe significant conversion of LC3-I to LC3-II following PGC-1 $\beta$ overexpression or downregulation (Fig. 5A). Autophagosome formation is characterized by a punctuated distribution of GFP-LC3. Cells were transiently transfected with GFP-LC3 and subcellular localization was detected by fluorescence microscopy. But we did not observed punctuated GFP fluorescence (Fig. 5B), suggesting that autophagy is not induced following PGC-1 $\beta$ expression.

We also measured the expression of Beclin 1, which is an essential autophagic protein $(26,27)$, and observed no change in the levels of Beclin 1 following PGC-1 $\beta$ exposure (Fig. 5A). Therefore, our results suggest that although mTORC1 is activated in MDA-MB-231 cells following pcDNA3-PGC-1 $\beta$ transfection, autophagy is not induced.

Inhibition of PGC-1 $\beta$ induced cell apoptosis. To evaluate the percentage of cell apoptosis induced by PGC-1 $\beta$, cells were stained with Annexin V-FITC and PI and analyzed by flow cytometry $24 \mathrm{~h}$ after pcDNA3-PGC-1 $\beta$ or pGenesilshPGC-1 $\beta$ exposure alone or in the presence of empty vector. We observed an increase in FITC positive cells, $24 \mathrm{~h}$ after pGenesil-shPGC-1 $\beta$ transfection, which was significantly reduced when the pcDNA3-PGC-1 $\beta$ was present (Fig. 6).

\section{Discussion}

In this study, we have shown that PGC-1 $\beta$-shRNA exposure in MDA-MB-231 cells affects mTOR signaling, thus modulating 
survival pathways, eventually causing cell death. We propose that PGC- $1 \beta$ initially affects the mTORC1 signaling complex. The increase in AMP levels leads to AMPK activation. We therefore hypothesize that AMPK activation induced by the increase in the AMP/ATP ratio following PAR synthesis inhibits mTORC1 in an attempt to preserve cellular energy.

The balance between survival and death signals is essential to cell fate. In our model, PGC-1 $\beta$ activation is rapid and intense and although at first, in an attempt to survive, activation of AMPK and inhibition of mTORC1 occur, the detrimental effects of free ADP-ribose polymers, free ADP-ribose and $\mathrm{NAD}^{+}$depletion overcome the beneficial effect of AMPK activation. Furthermore, our results suggest that mitochondria production contributes to PGC-1 $\beta$ activation (Fig. 2B). After exposure to PGC-1 $\beta$, the Beclin 1, LC3-I and LC3-II modulation was not changed (Fig. 5).

Previous studies have shown that the PGC-1 family of coactivators is a potent stimulator of mitochondrial respiration and gene transcription in liver, heart, and skeletal muscle $(28,29)$. It has been shown that PGC-1 acts by activating the NRF1 and NRF2 that in turn regulate expression of mtTFA, essential for replication, maintenance, and transcription of mitochondrial DNA (30). PGC-1 is also important for the expression of nuclear genes encoding respiratory chain subunits and other proteins that are required for proper mitochondrial functions (31). Our studies demonstrate that PGC-1 $\beta$ activation may trigger apoptotic responses, depending on cell type and functional signaling pathways. Tumorigenesis is a complex, multistep process characterized by the dysregulation of many signaling cascades, including the mTOR signaling pathway (32). Many of mTOR's upstream regulators and downstream effectors are aberrantly activated in different types of human cancer, heightening interest in mTOR signaling. Because the malignant phenotype depends on these signaling proteins, it is not surprising that mTOR is viewed as a potential target for cancer therapy. Therefore, various approaches to inhibiting the mTOR signaling pathway are being pursued for clinical development $(33,34)$.

It has been shown that phosphorylation of Raptor by AMPK induces a metabolic check-point to inhibit cell growth (23). Activation of AMPK and subsequent suppression of mTORC1 activity can also induce a cytoprotective autophagic response $(35,36)$. In our model, we observed AMPK activation and Raptor phosphorylation following PGC-1 $\beta$ overexpression (Fig. 4A and C).

Autophagy is an apoptosis-alternative pathway to induce cell death, PGC-1 $\beta$-shRNA can downregulate the AMPK activation and induce tumor cell apoptosis, but can not affect autophagy. Our study showed the change of autophagic competent LC3-II did not occur with or without PGC-1 $\beta$ (Fig. 5A). In addition, the lack of formation of a punctuate signal of GFP-LC3 transiently transfected in cancer cells (Fig. 5B), and the unaltered Beclin 1 expression (Fig. 5A), indicate that autophagy is not induced.

In conclusion, these results provide new evidence that cell apoptosis is orchestrated by the balance between several signaling pathways. PGC-1 $\beta$ downregulation by shRNA leads to a decrease in the expression of mTOR pathway-related genes in MDA-MB-231 cells, and PGC-1 $\beta$ induced the cell apoptosis mediated by mTOR signal pathway.

\section{Acknowledgements}

This study was supported by the National Nature Science Foundation of China (NSFC, grant no. 81000958). The funders had no role in study design, data collection and analysis, decision to publish, or preparation of the manuscript. We thank Dr Shihai Liu for his technical assistance. We also thank Drs Xi Yang, Hua Zhou and Shihai Liu for critical reading of this manuscript.

\section{References}

1. Ginsburg OM and Love RR: Breast cancer: a neglected disease for the majority of affected women worldwide. Breast J 17: 289-295, 2011.

2. Lorico A and Rappa G: Phenotypic heterogeneity of breast cancer stem cells. J Oncol 2011: 135039, 2011.

3. Misra Y, Bentley PA, Bond JP, Tighe S, Hunter T and Zhao FQ: Mammary gland morphological and gene expression changes underlying pregnancy protection of breast cancer tumorigenesis. Physiol Genomics 44: 76-88, 2012.

4. Bray F, McCarron P and Parkin DM: The changing global patterns of female breast cancer incidence and mortality. Breast Cancer Res 6: 229-239, 2004.

5. Perou CM: Molecular stratification of triple-negative breast cancers. Oncologist 16 (Suppl 1): S61-S70, 2011.

6. Liu S and Wicha MS: Targeting breast cancer stem cells. J Clin Oncol 28: 4006-4401, 2010.

7. Tremblay AM and Giguère V: The NR3B subgroup: an ovERRview. Nucl Recept Signal 5: 1-11, 2007.

8. Hartlerode A, Odate S, Shim I, Brown J and Scully R: Cell cycledependent induction of homologous recombination by a tightly regulated I-SceI fusion protein. PloS One 6: e16501, 2011.

9. Vezina C, Kudelski A and Sehgal SN: Rapamycin (AY-22,989), a new antifungal antibiotic. I. Taxonomy of the producing streptomycete and isolation of the active principle. J Antibiot (Tokyo) 28: 721-726, 1975.

10. Cunningham JT, Rodgers JT, Arlow DH, Vazquez F, Mootha VK and Puigserver P: mTOR controls mitochondrial oxidative function through a YY1-PGC-1alpha transcriptional complex. Nature 450: 736-740, 2007

11. Kelly DP and Scarpulla RC: Transcriptional regulatory circuits controlling mitochondrial biogenesis and function. Genes Dev 18: 357-368, 2004.

12. Spiegelman BM: Transcriptional control of mitochondrial energy metabolism through the PGC1 coactivators. Novartis Found Symp 287: 60-69, 2007.

13. Arany Z, Lebrasseur N, Morris C, et al: The transcriptional coactivator PGC-1beta drives the formation of oxidative type IIX fibers in skeletal muscle. Cell Metab 5: 35-46, 2007.

14. Czubryt MP, McAnally J, Fishman GI and Olson EN: Regulation of peroxisome proliferator-activated receptor gamma coactivator 1 alpha (PGC-1 alpha) and mitochondrial function by MEF2 and HDAC5. Proc Natl Acad Sci USA 100: 1711-1716, 2003.

15. Attia RR, Connnaughton S, Boone LR, et al: Regulation of pyruvate dehydrogenase kinase 4 (PDK4) by thyroid hormone: role of the peroxisome proliferator-activated receptor gamma coactivator (PGC-1 alpha). J Biol Chem 285: 2375-2385, 2010.

16. Schieke SM, Phillips D, McCoy JP Jr, et al: The mammalian target of rapamycin (mTOR) pathway regulates mitochondrial oxygen consumption and oxidative capacity. J Biol Chem 281: 27643-27652, 2006.

17. Ramanathan A and Schreiber SL: Direct control of mitochondrial function by mTOR. Proc Natl Acad Sci USA 106: 22229-22232, 2009.

18. Wu Z, Puigserver P, Andersson U, et al: Mechanisms controlling mitochondrial biogenesis and respiration through the thermogenic coactivator PGC-1. Cell 98: 115-124, 1999.

19. St-Pierre J, Lin J, Krauss S, et al: Bioenergetic analysis of peroxisome proliferator-activated receptor gamma coactivators 1alpha and 1beta (PGC-1alpha and PGC-1 $\beta$ ) in muscle cells. J Biol Chem 278: 26597-26603, 2003.

20. Hardie DG: Minireview: the AMP-activated protein kinase cascade: the key sensor of cellular energy status. Endocrinology 144: 5179-5183, 2003. 
21. Kemp BE, Stapleton D, Campbell DJ, et al: AMP-activated protein kinase, super metabolic regulator. Biochem Soc Trans 31: $162-168,2003$

22. Willer A: Reduction of the individual cancer risk by physical exercise. Onkologie 26: 283-289, 2003.

23. Gwinn DM, Shackelford DB, Egan DF, et al: AMPK phosphorylation of raptor mediates a metabolic checkpoint. Mol Cell 30: 214-226, 2008

25. Kabeya Y, Mizushima N, Ueno T, et al: LC3, a mammalian homologue of yeast Apg8p, is localized in autophagosome membranes after processing. EMBO J 19: 5720-5728, 2000.

24. Hardie DG, Salt IP, Hawley SA and Davies SP: AMP-activated protein kinase: an ultrasensitive system for monitoring cellular energy charge. Biochem J 338: 717-722, 1999.

26. Cao Y and Klionsky DJ: Physiological functions of Atg6/ Beclin 1: a unique autophagy-related protein. Cell Res 17: 839-849, 2007.

27. Pattingre S, Espert L, Biard-Piechaczyk M and Codogno P: Regulation of macroautophagy by mTOR and Beclin 1 complexes. Biochimie 90: 313-323, 2008.

28. Meirhaeghe A, Crowley V, Lenaghan C, et al: Characterization of the human, mouse and rat PGC1 beta (peroxisome-proliferatoractivated receptor-gamma co-activator 1 beta) gene in vitro and in vivo. Biochem J 373: 155-165, 2003.

29. Lin J, Tarr PT, Yang R, et al: PGC-1ßeta in the regulation of hepatic glucose and energy metabolism. J Biol Chem 278: 30843-30848, 2003.
30. Finck BN and Kelly DP: PGC-1 coactivators: inducible regulators of energy metabolism in health and disease. J Clin Invest 116: 615-622, 2006.

31. Feige JN and Auwerx J: Transcriptional coregulators in the control of energy homeostasis. Trends Cell Biol 17: 292-301, 2007.

32. Peralba JM, DeGraffenried L, Friedrichs W, et al: Pharmacodynamic evaluation of CCI-779, an inhibitor of mTOR, in cancer patients. Clin Cancer Res 9: 2887-2892, 2003.

33. Boulay A, Zumstein-Mecker S, Stephan C, et al: Antitumor efficacy of intermittent treatment schedules with the rapamycin derivative RAD001 correlates with prolonged inactivation of ribosomal protein S6 kinase 1 in peripheral blood mononuclear cells. Cancer Res 64: 252-261, 2004.

34. Basso AD, Mirza A, Liu G, Long BJ, Bishop WR and Kirschmeier P: The farnesyl transferase inhibitor (FTI) SCH66336 (lonafarnib) inhibits Rheb farnesylation and mTOR signaling. Role in FTI enhancement of taxane and tamoxifen anti-tumor activity. J Biol Chem 280: 31101-31108, 2005.

35. Harhaji-Trajkovic L, Vilimanovich U, Kravic-Stevovic T, Bumbasirevic V and Trajkovic V: AMPK-mediated autophagy inhibits apoptosis in cisplatin-treated tumour cells. J Cell Mol Med 13: 3644-3654, 2009.

36. Herrero-Martin G, Høyer-Hansen M, Garcia-Garcia C, et al: TAK1 activates AMPK-dependent cytoprotective autophagy in TRAIL-treated epithelial cells. EMBO J 28: 677-685, 2009. 